\title{
PARTITIONING OF POWER IN FLAT ROLLING
}

\author{
Mohamed Khaled, Ramy Mohamed and Mazen Negm \\ Mechanical Engineering Department, Faculty of Engineering, Al-Azhar University
}

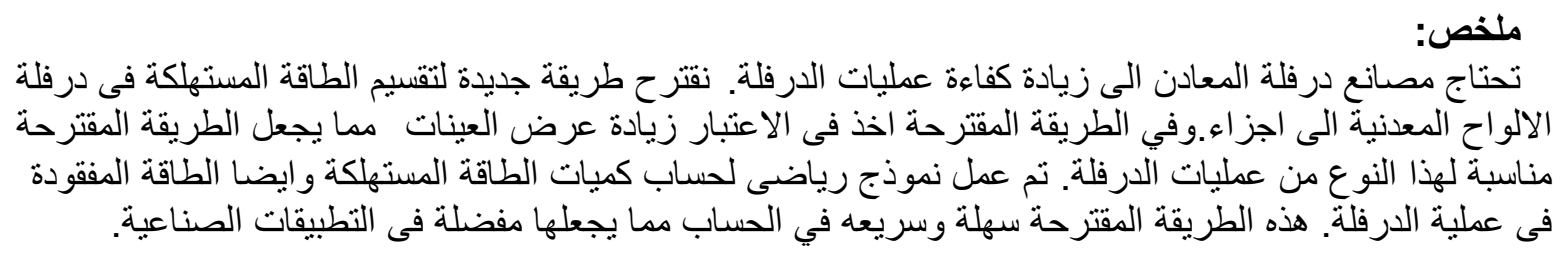

\begin{abstract}
The metal rolling industry is in continuous demand for increasing the efficiency of the process. A new method of partitioning the consumed power in plate rolling into its constituent parts is presented. The proposed model takes into consideration the spread of workpiece, making it applicable to more general situations where the height to length ratio is equal to or larger than one. A mathematical model is proposed to calculate the consumed power and calculate different losses in the rolling operation. The method is easy and computationally efficient, which makes it attractive to industrial application.
\end{abstract}

\section{Key Words: Rolling, Power, Mathematical Model.}

\section{INTRODUCTION}

Rolling is one of the oldest processes used in the metal working industry. In view of the tremendous volume and wide variety of rolled products manufactured each year, rolling -is considered one of the most important forming processes-which received many research efforts. The present work addresses the cold rolling of plates with aspect ratio equal to or larger than one.

The objective of the flat rolling process is to reduce the thickness of the workpiece from an initial thickness to a pre-determined final thickness. This is typically accomplished on a rolling mill, in which two work-rolls, rotating in opposite directions, draw the plate to be rolled into the roll gap and forcing it through to the exit, causing the required reduction of the thickness [1]. The process is illustrated in Fig. 1.

Several attempts to measure the pressure distribution between the rolls and workpiece during rolling were reported in literature. Siebel [2] was among the first to attempt to measure this quantity using a large $(2 \times 2 \mathrm{~mm})$ pressure pin. The homogeneous deformation method proposed requires the selection of an element in the workpiece and identifying all normal and frictional forces acting on this element. The principal assumption is that the deformation of metal is homogenous throughout the whole deformation zone [3]. 


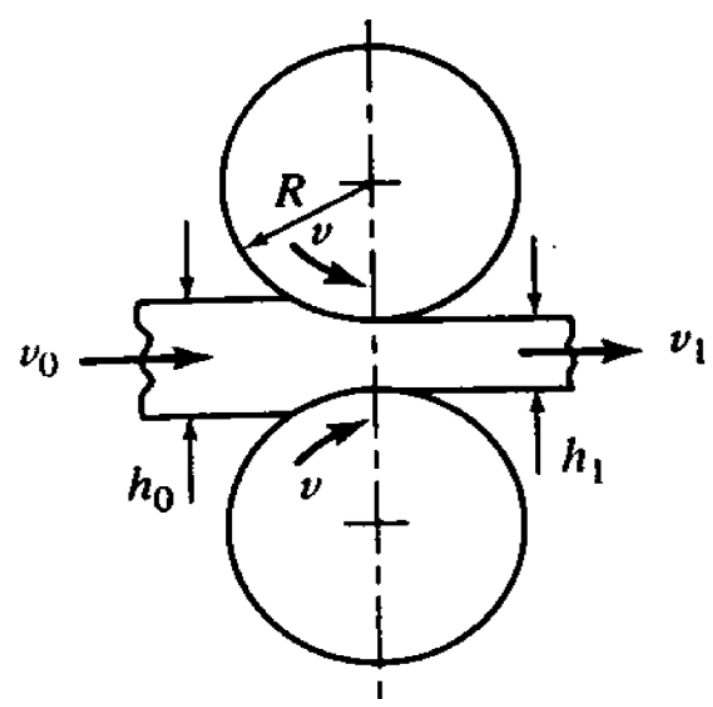

Figure 1: work piece from the initial thickness to a pre-determined final thickness [4].

The homogenous deformation method does not take into account the reversal in direction of the shear stresses since the effect of frictional forces is included as a scalar correction factor. Von Karman proposed a one-dimensional model for the rolling process, in which he neglected the spread in width. the ordinary differential equation describing the pressure distribution was established from a consideration of the equilibrium of forces acting on the elemental slab in the deformation zone. Hence, the method is typically referred to as the "slab method" [5].

Orowan's model [6] and [7] considered to be the most comprehensive. Where the graphical integration techniques to solve Von Karman's equation. Sims [8] modified Orowan's model by many simplifying assumptions in order to obtain a closed form solution of the equilibrium equation. Alexander [9], [10] greatly simplified the use of the Von Karman equation by presenting a computer-aided solution. He obtained more accurate results than the then existing homogeneous theories.

Since 1980 , finite element models have been used as an effective tool for studying the rolling process by solving numerically the governing differential equations describing the three-dimensional deformation behavior of the workpiece. The finite element method do provide more information with better accuracy than simplified analytic methods. The drawback is its massive computational requirements, especially in three-dimensional analysis. In general, it requires a huge CPU time and memory resources because of complicated adaptive remeshing, adaptive time stepping due to the nature of nonlinear convergence and treatment of boundary conditions. These factors made the use of FEM in on-line analysis of rolling force and pressure very difficult.

Therefore, to achieve rapid analysis applicable to on-line control of the operation it is required to develop an approximate model to catch the most important characteristics of the process. The slab method has been successfully applied to plate and strip rolling. Since the slab method is based on the plane strain assumption, it may yield considerable inaccuracies when applied to processes in which the spread of width cannot be neglected. Thus, it is highly desirable to develop a more effective method for calculating roll force, and the deformation power when the spread of width during rolling need to be considered.

In the present work, a model for the partitioning of power as well as rolling force is proposed. The main motivation is to match the observables in the industrial context i.e. the measured motor power. The power losses can be fitted to measured motor electrical power after exclusion of the power consumed in the deformation. 


\section{EXPERIMENTAL WORK}

The rolling machine designed and manufactured to be used in the present work is shown in Fig. 2. The dimensions of the working roller and some of the relevant parameters are listed below:

- diameter of roller $120 \mathrm{~mm}$,

- $\mathrm{AC}$ motor $(3 \mathrm{~kW})$ with speed $1420 \mathrm{rpm}$, and

- gear box with a speed reduction ratio 1420/33.

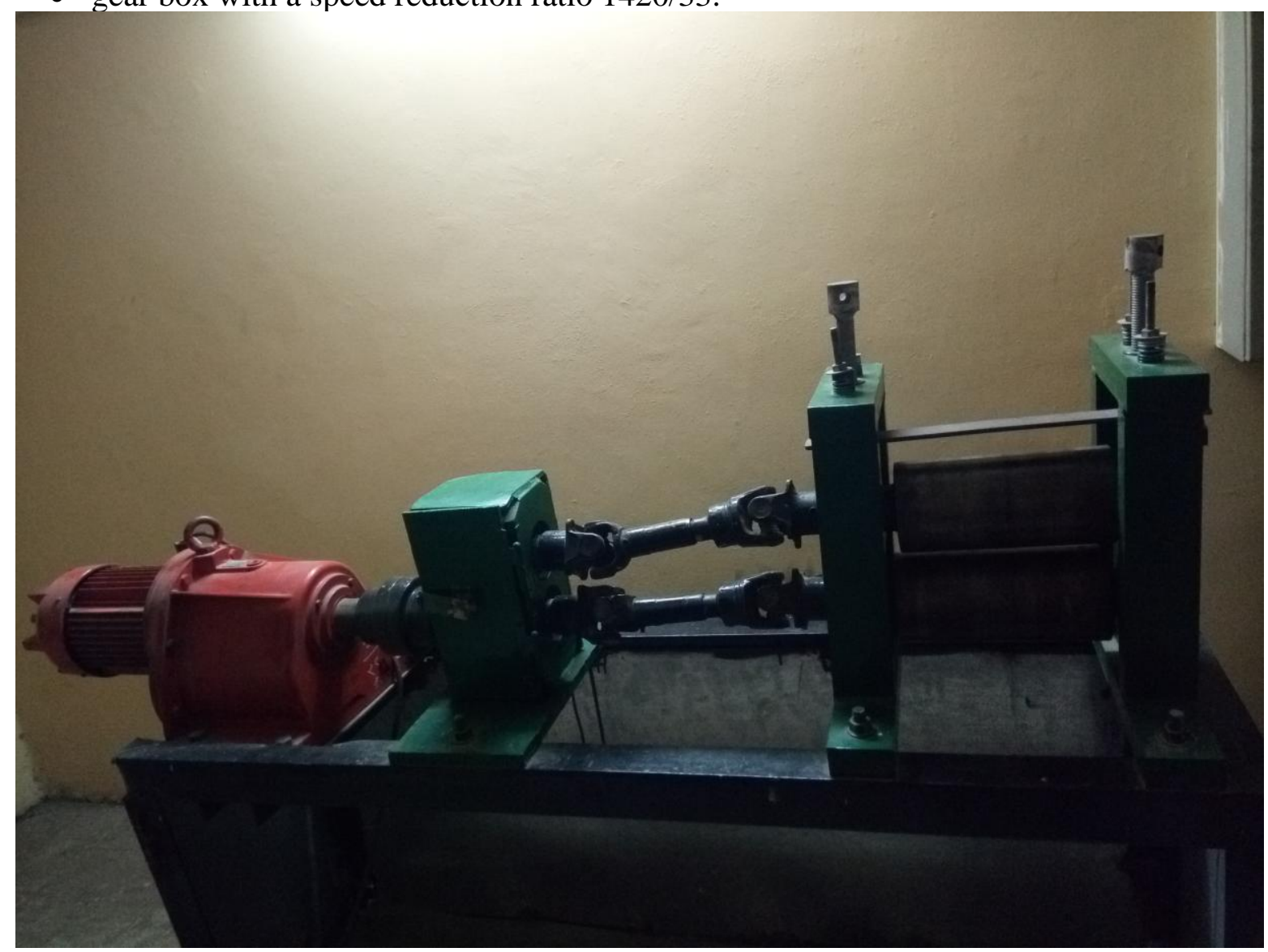

Figure 2: The rolling machine used in the experimental work.

The material used as a stock was brass alloy in the form of a flat bar with dimensions $12.73 \times 12.73 \mathrm{~mm}$. The cold rolling process starts after preparation of the sample and adjusting the gap between two roller by using a filler. The motor power was measured by using clamp wattmeter that measures the total power consumed during the process. This process was repeated for different drafts by changing the gap between the two rolls and measuring the input power to the motor during the process.

After each rolling pass, a sample was cut parallel to the direction of the rolling, and a standard tension test specimen was prepared in order to evaluate the yield strength of the material and the work hardening behavior. For each rolling pass with a specific draft, two tension samples were prepared and the average of the yield strength measured was calculated. Those average values are the ones that are substituted in the mathematical model later.

\section{MATHEMATICAL MODEL}

The power supplied by the motor shaft to drive the rolls of rolling mill could be partitioned into the following components:

Where $P_{r}$ is the power needed to achieve the plastic deformation of the rolled part, $P_{f}$ is the additional power needed to overcome the frictional forces appearing during rolling in 
the mechanical components of transmission. $P_{\text {idle }}$ is the torque of idle running (i.e. running the machine without rolling), and $P_{\text {dynamic }}$ is the dynamic component loading the motor shaft in order to overcome the inertial forces due to non-uniform rotation of the rolls. Since the power partitioning at the steady state, is of main increase the dynamic component will be neglected.

\subsection{Deformation geometry}

A schematic representation of the deformation geometry of the workpiece-rolling process is shown in Fig. 3. Cartesian coordinates $x, y, z$ are chosen to be the directions of workpiece length, height and width respectively, with the origin of axes at the midpoint of the entry plane.

The $x-y$ and $y-z$ planes are planes of symmetry; $2 h_{o}, 2 h_{1}$ are the initial, final heights and $2 h_{x}$ is the height at distance $x$ and $2 b_{o}, 2 b_{1}$ are the initial and final widths and $2 b_{x}$ is the width at distance $x$ from the entry plane.

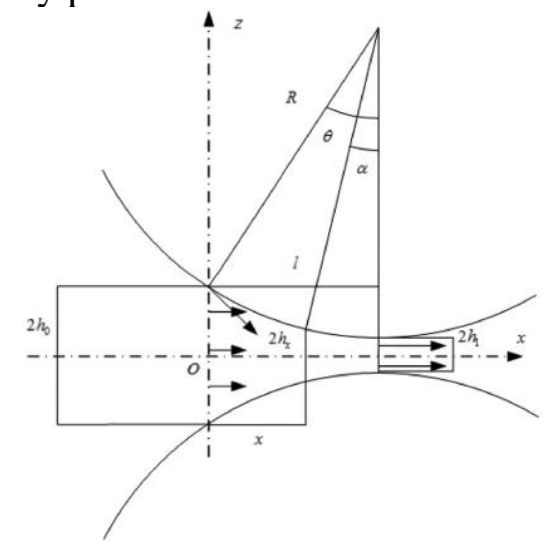

Figure 3: Cross-sectional plane of the rolling process [13].

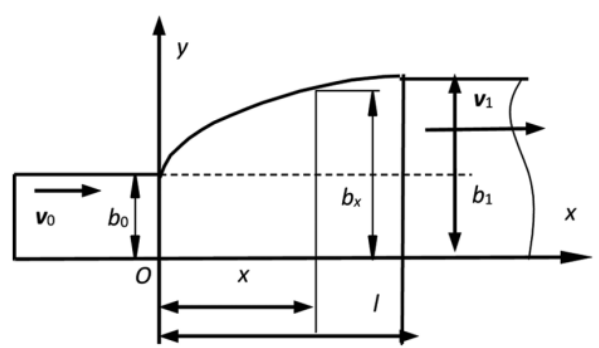

Figure 4: Half section of $x-y$.

To describe draft and spread deformation shown figure 4, the projected length of the roll bite $L$ may be expressed as a function of the geometric variables, i.e. $\quad L=R \sin \theta=R \sqrt{1-\cos ^{2} \theta}$

The equation for biting angle is given as

$$
\cos \theta=\frac{R-\Delta h}{R}
$$

Where $R$ is the roll radius and the roll is assumed to be rigid, and the draft is defined as

$$
\Delta h=h_{o}-h_{1}
$$

Substituting Eq. (2) into the projected length of the roll bite and considering that the draft $\Delta h$ is much smaller than $R$ results in

The equations of the

$$
L=\sqrt{2 R \Delta h}
$$




$$
\begin{gathered}
h_{x}=R+h_{1}+\sqrt{R^{2}-(L-x)^{2}} \\
b_{x}=b_{1}-\frac{\Delta b(L-x)^{2}}{L^{2}}
\end{gathered}
$$

And we define $b_{m}=\left(b_{o}+2 b_{m}\right) / 3$ as in [13].

\subsection{The power of deformation}

Wang et al. [13] suggested the mathematical expression of the equal perimeter EP yield criterion and the derivation of the specific plastic work rate. The mathematical expression of the EP yield criterion in the Haigh-Westergaard stress space:

$$
\begin{aligned}
\sigma_{1}-\frac{1}{4} \sigma_{2}-\frac{3}{4} \sigma_{3} & =\sigma_{y} \text { if } \sigma_{2} \leq \frac{1}{2}\left(\sigma_{1}+\sigma_{2}\right) \\
\frac{3}{4} \sigma_{1}+\frac{1}{4} \sigma_{2}-\sigma_{3} & =\sigma_{y} \text { if } \sigma_{2} \geq \frac{1}{2}\left(\sigma_{1}+\sigma_{2}\right)
\end{aligned}
$$

The geometric representation of the yield criterion on the $\pi$-plane is equilateral but nonequiangular as shown in Figure 5.

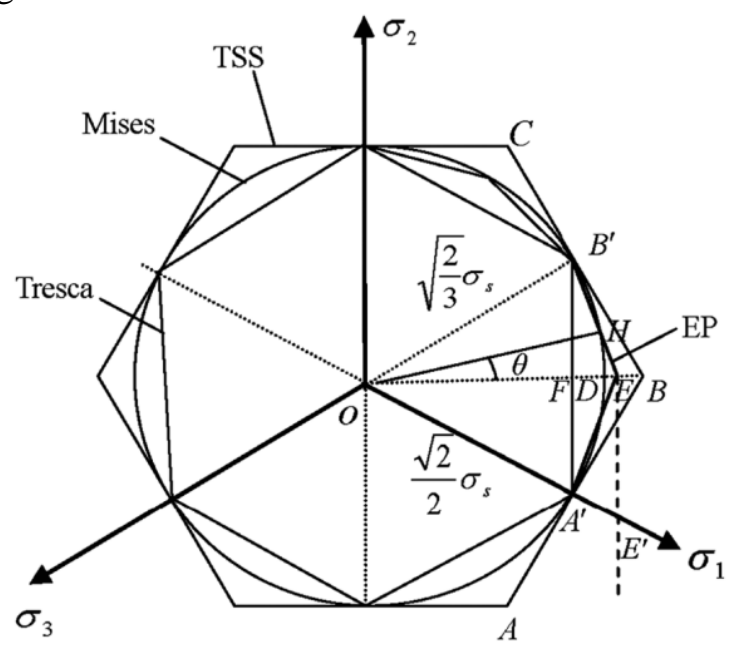

Figure 5: Yield loci on the $\pi$-plane.

According to Levy-Mises flow rule, the specific power of plastic deformation of EP yield criterion could be written:

$$
D\left(\dot{\epsilon}_{i j}\right)=0.5897 \sigma_{y}\left(\dot{\epsilon}_{\max }-\dot{\epsilon}_{\min }\right)
$$

From equation (7) it is noted that the specific plastic power is a linear function of the yield strength of the material, the maximum and the minimum strain rate. This linearity is the cornerstone for the direct evaluation of the power.

A kinematic admissible velocity field typically postulated as:

$$
\begin{aligned}
& v_{x}=v_{o}\left(1+\lambda \frac{h_{o}-h_{x}}{h_{x}}\right) \\
& v_{y}=v_{o}(\lambda-1) y \frac{1}{h_{x}} \frac{d h_{x}}{d x} \\
& v_{z}=v_{o}\left(1+\lambda \frac{h_{o}-h_{x}}{h_{x}}\right) z \frac{1}{h_{x}} \frac{d h_{x}}{d x}
\end{aligned}
$$


Where the value of $\lambda$ can be obtained according to the conservation of mass as:

$$
\lambda=1-\frac{\Delta b h_{o}}{\Delta h b_{m}}
$$

It was assumed that the workpiece exits the roll bite with velocity, $v_{1}=\omega R$ where $\omega$ is the roll angular velocity. So, in the present work the forward was not considered.

The rolling power Pr in Eq. (1) should be decomposed into two components, the part that provides the work required for achieving the preset plastic deformation $\mathrm{Pp}$, and the part to compensate for the frictional losses between the roll and the workpiece being rolled due to velocity difference between the roll and the workpiece .

The power of plastic deformation can be computed by integrating Eq. (7) with respect to volume, to give:

$$
P_{p}=2.3588 \sigma_{y} v_{o} b_{o}\left((1-\lambda) \Delta h+2 \lambda h_{o} \ln \frac{h_{o}}{h_{1}}\right)
$$

Note that this equation is missing the power consumed in overcoming the friction losses due to velocity difference between the roll and the workpiece $P_{\tau}$.

It is then assumed that

$$
P_{r}=P_{p}+\mu P_{\tau}
$$

In order to approximate the value of the $P_{\tau}$ and considering that forward slip is neglected (i.e. the neutral angle is the deformation angle). The frictional losses can be described as:

$$
P_{\tau}=4 \frac{\sigma_{y}}{\sqrt{3}} R v_{r} b_{m} \theta
$$

where $\mu$ is Coulomb friction coefficient, and $\theta$ is the angle subtending the plastic deformation zone [14].

Our main assumption is that the frictional losses during rolling in the transmission and bearings are proportional to the power corresponding to the separating force $F_{s} \bar{v}_{z}$.

The separating force is computed from the torque corresponding to the plastic deformation torque,

$$
F_{S}=\frac{P_{p}}{\omega_{r} \chi L}
$$

Where the arm factor $\chi$ refer to the following equation:

$$
\chi=0.39+0.295 \exp \left(-0.193 \frac{R}{2 h_{m}}\right)
$$

And $h_{m}$ is defined as:

$$
h_{m}=h_{1}+\frac{\Delta h}{2}-\frac{R^{2} \theta}{2 L}
$$

In the exit section of the deformation zone, the velocity in the $\mathrm{z}$ direction equals zero, and since we are neglecting the forward slip, it is sufficient to consider the velocity in the entry section. Here, it is considered that if the double barreling in plate rough rolling occurs, then a parabolic distribution of $\mathrm{vz}$ along $\mathrm{z}$-direction would be a valid assumption. Thus, on the entry section, the following average velocity in the $\mathrm{z}$-direction as: 


$$
\bar{v}_{z}=\frac{v_{o} \tan \theta}{3}
$$

So, the final power partition that is suggested:

$$
P_{m}-P_{\text {idle }}-P_{r}=\mu P_{\tau}+A F_{s} \bar{v}_{z}
$$

This model represents an over-determined system, provided that the number of measurements of $P_{m}$ are more than two, the unknowns in the model are $\mu$ and $A$. The system of equations is formulated as a linear system, which are then solved using least squares method.

\section{RESULTS AND DISCUSSION}

In figure (6) the relationship between the experimentally measured motor power minus the idle power and friction losses in plastic deformation power $\mu \mathrm{P} \tau$. The fiction losses are evaluated based on the value of the friction coefficient solved from the Eq. (7). The friction losses are plotted versus the different values of rolling draft used in the experimental study. The general trend of increasing losses are witnessed, this may be attributed to the increase in the displaced volume of material as the draft increases.

In figure 7 the relationship between the motor power measured and predicted friction losses in transmission $A F_{s} \bar{v}_{z}$ due to rolling as a function of rolling draft. The power friction losses in transmission due to rolling is less than the friction losses in plastic deformation power, also friction losses in transmission due to rolling is almost constant with the different values of draft.

Figure 8 shows the relationship between the motor power measured and plastic deformation power calculated from Eq. (11) with the experimentally measured geometrical values as a function of the draft.

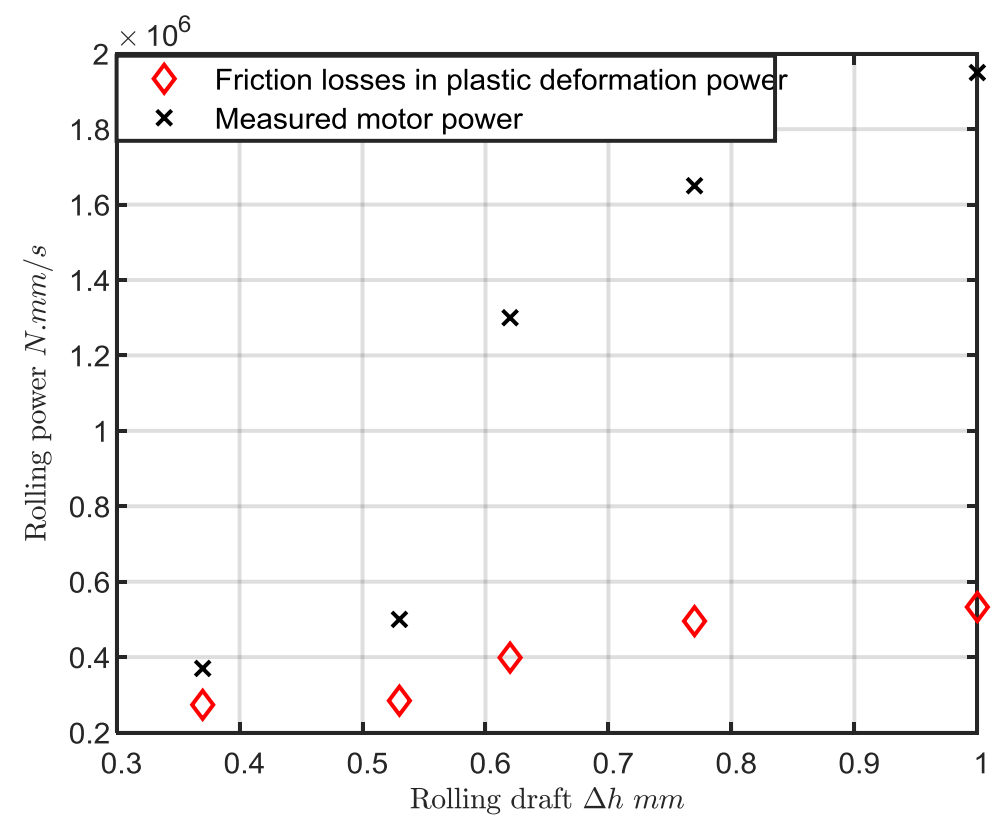

Figure 6: The proposed model of friction losses in plastic deformation power compared with the measured motor power - idle power.

Figure 9 shows the relationship between the motor power measured and total predicted power due to rolling (the sum of powers). The predicted power includes the plastic deformation power, friction losses in plastic deformation power and the power friction losses in transmission due to rolling. The overly predicted values of the power in the small draft are an artifact of the linearity assumption. 


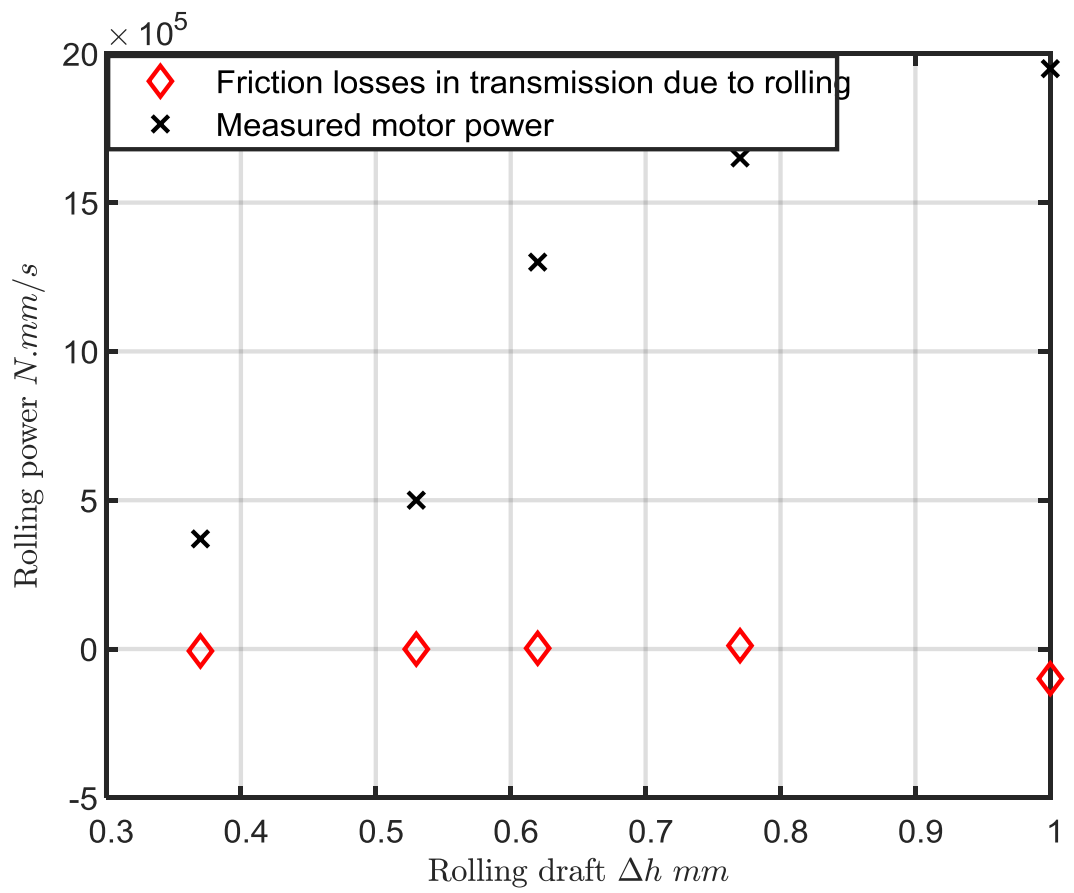

Figure 7: the proposed model of power friction losses in transmission due to rolling compared with the measured motor power - idle power.

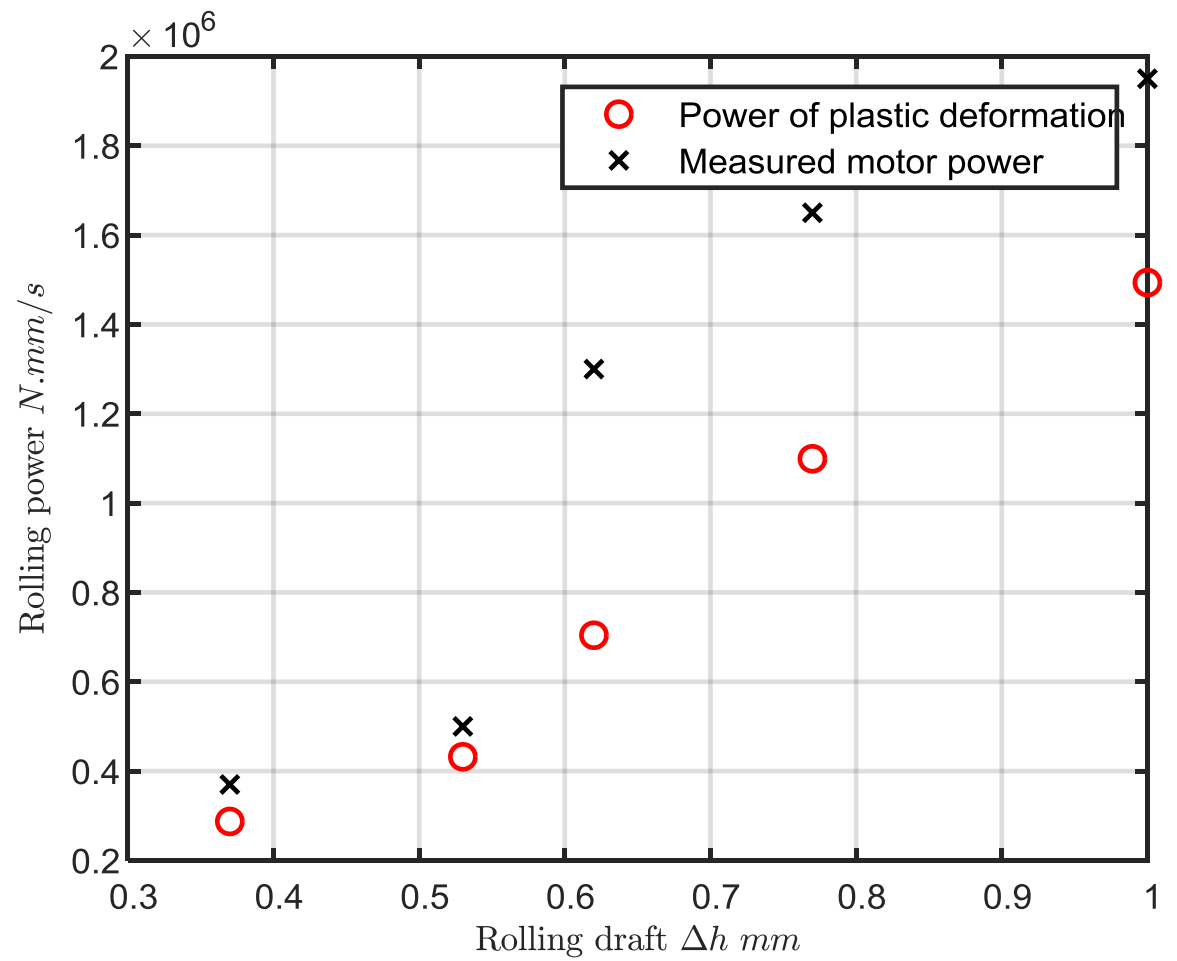

Figure 8: The proposed model of power of plastic deformation compared with the measured motor power - idle power. 


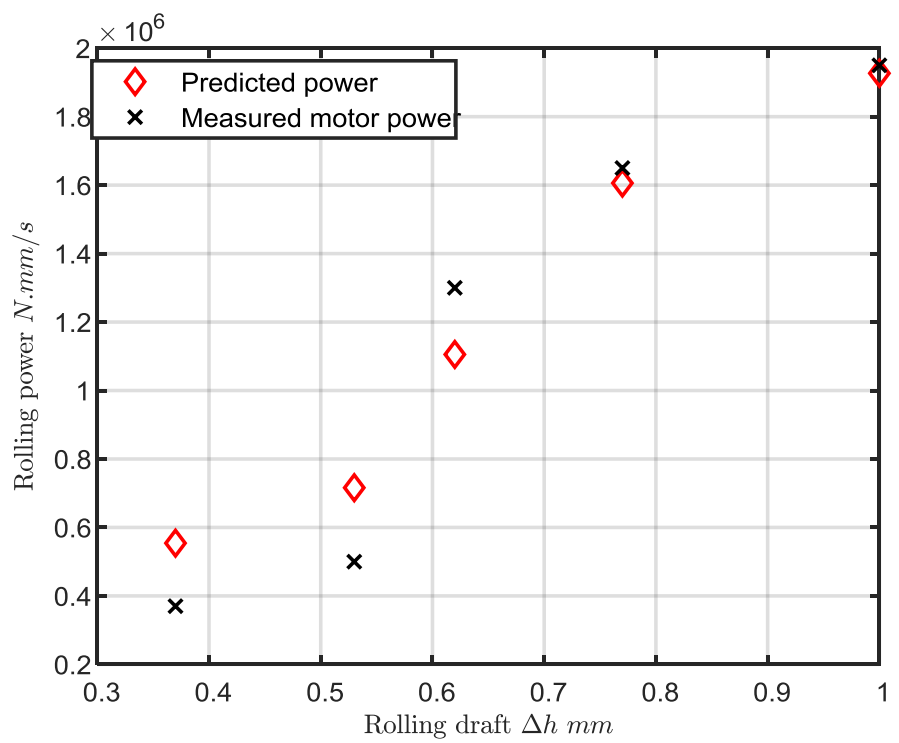

Figure 9: The proposed model of predicted power compared with the measured motor power - idle

\section{CONCLUSION}

power.

The predictive tenets of the proposed model have been demonstrated. The value of the model in the industrial setting, where the main measurable quantity is the motor power, for servicing and online control are the major motivation for the present work. The linearity of the model, and the simplifying assumptions regarding the exit and entry velocity may not be generalizable, which would be addressed in future work.

\section{REFERENCES}

[1] V. B. Ginzburg and R. Ballas, Flat rolling fundamentals, 1st ed. CRC, 2000.

[2] E. G. Thomsen, C. T. Yang, and S. Kobayashi, Mechanics of Plastic Deformation In Metal Processing. McMillan, 1965.

[3] D. Jortner, J. F. Osterle, and C. F. Zorowski, "An analysis of cold strip rolling," Int. J. Mech. Sci., vol. 2, pp. 179-194, 1960.

[4] J. G. Lenard, Primer on Flat Rolling, 2nd ed. Elsevier, 2014.

[5] F. A. R. Al-Salehi, T. C. Firbank, and P. R. Lancaster, "An experimental determination of the roll pressure distributions in cold rolling," Int. J. Mech. Sci., vol. 15, no. 9, pp. 693-710, 1973.

[6] E. Orowan, "The Calculation of Roll Pressure in Hot and Cold Flat Rolling," Proc. Inst. Mech. Eng., vol. 150, pp. 140-167, 1943.

[7] E. Orowan, "Communications on The Calculation of Roll Pressure in Hot and Cold Flat Rolling," Proc. Inst. Mech. Eng., vol. 150, pp. 314-324, 1943.

[8] D. R. Bland and R. B. Sims, "A Note on the Theory of Rolling with Tensions," pp. 371-374, 1952.

[9] J. M. Alexander, "On the Theory of Rolling," Proc. R. Soc. A Math. Phys. Eng. Sci., vol. 329, no. 1579, pp. 493-496, 1972.

[10] J. M. Alexander, "Corrigenda: On the Theory of Rolling," Proc. R. Soc. A Math. Phys. Eng. Sci., vol. 329, no. 1579, pp. 493-496, 1972.

[11] K. Devarajan, K. P. Marimuthu, and A. Ramesh, "FEM Analysis of Effect of Rolling Parameters on Cold Rolling Process," vol. 2, no. 1, pp. 35-40, 2012.

[12] H. S. Valberg, Applied Metal Forming: Including Fem Analysis. Cambridge University Press, 2010.

[13] L. Wang, X. Zhu, D. Zhao, and D. Zhang, "An analytical model of plate rolling force with a simple available velocity field and equal perimeter yield criterion," $A d v$. Mech. Eng., vol. 9, no. 9, p. 168781401772883, 2017.

[14] J. G. Lenard, Primer on flat rolling, 1st ed. Elsevier, 2007. 